\title{
"Words, words, words": théâtralité et aporie du langage dans
} Richard II

Marie-Christine Munoz

\section{Citer ce document / Cite this document :}

Munoz Marie-Christine. "Words, words, words": théâtralité et aporie du langage dans Richard II. In: XVII-XVIII. Bulletin de la société d'études anglo-américaines des XVIle et XVIIle siècles. N59, 2004. pp. 77-93;

doi : https://doi.org/10.3406/xvii.2004.1996

https://www.persee.fr/doc/xvii_0291-3798_2004_num_59_1_1996

Fichier pdf généré le 27/03/2018 


\section{"WORDS, WORDS, WORDS": \\ POLITIQUE, THÉATRALITÉ ET APORIE DU LANGAGE \\ DANS RICHARD $\boldsymbol{I}$}

Four lagging winters and four wanton springs End in a word: such is the breath of kings.

(Richard II, 1.3.214-215)

Au tout début de son étude sur les vertus du mode poétique, Sir Philip Sidney appuie son argumentation sur la définition aristotélicienne de la nature mimétique de la représentation poétique. Il met en avant les impératifs de persuasion qui s'imposent au poète et souligne l'importance fondatrice du principe de plaisir dans la réception de l'œuvre poétique: "Poesie therefore, is an Art of Imitation: for so Aristotle termeth it in the word $\mu \iota \mu \eta \sigma \imath \varsigma$, that is to say, a representing, counterfeiting, or figuring forth to speake Metaphorically. A speaking Picture, with this end to teach and delight." ${ }^{1}$ Ces attributs essentiels font l'unanimité des auteurs de traités sur le mode littéraire le plus noble durant la Renaissance anglaise. George Puttenham, dans son Arte of English Poesie recourt à une analogie musicale afin d'illustrer la force persuasive d'un mode d'expression qui fonde son efficacité sur le plaisir qu'il génère chez son destinataire. ${ }^{2}$ Tous s'accordent à louer l'aptitude notable à l'éloquence du mode poétique, qui donne bien entendu sa pleine mesure au théâtre, tout particulièrement lorsque le dramaturge fait du fonctionnement langagier son intérêt premier. Il en va ainsi du drame du roi Richard II, que Shakespeare compose en remodelant diverses sources, au nombre desquelles nous pouvons compter The Third Volume of Chronicles par Raphael Holinshed (édition de 1587), The First Fowre Bookes Of The Civile Wars Between

1. Sir Philip Sidney, The Defence of Poesie, Political Discourses, Correspondence, Translations, ed. Albert Feuillerat (Cambridge: Cambridge UP, 1923) 9.

2. George Puttenham, The Arte of English Poesie (London, 1589), STC 20519. 
The Two Houses of Lancaster and Yorke de Samuel Daniel (1595), et Woodstock de la main d'un anonyme.

Quatre siècles plus tard, une large part de la critique shakespearienne reconnaît l'intérêt fondamental d'une étude des représentations du politique dans les pièces historiques, à la lumière des enjeux linguistiques du texte. Richard II suscite alors une attention toute particulière, en raison de la place centrale qu'occupe la réflexion sur le langage dans cette pièce. Ces lecteurs avertis du texte shakespearien s'accordent à identifier en la figure du souverain déposé un "Prince des mots," qui distille habilement la parole et dont la chute peut se confondre avec un anéantissement linguistique. Une aporie langagière, en somme. C'est ainsi que l'on trouve sous la plume de critiques qui font autorité, au nombre desquels figure en bonne place Wolfgang Clemen, une analyse de la logorrhée du roi en des termes particulièrement catégoriques. Il est question de "this tendency of the king to give himself away to words," ou encore de "Richard's propensity for verbalizing," et d'une "attitude towards words which serve him as a sort of substitute for reality." ${ }^{3}$ Richard, le roi poc̀te, Richard, le roi acteur, tels sont les visages de cette figure polymorphe mise à nu par une critique avide de justifier la présence obsédante du corset rhétorique qui gaine le drame du roi déposé. Rhétorique qui apparaît désormais comme un substitut mystificateur que le monarque incompétent dans le domaine politique préfère à l'établissement sans appel de son pouvoir dans l'action. Mark Van Doren revendique avec conviction cette lecture dans laquelle Leonard Tennenhouse reconnaît une volonté de dissocier signe et pouvoir, ou plutôt de concevoir le signe linguistique comme un pis-aller, un pic̀tre substitut au pouvoir royal véritablement exercé, la pitoyable tentative d'un monarque acculé pour masquer son incapacité à exercer l'autorité: "Richard has been called the poet king by critics who want to read him in the nineteenth century manner, as a poet king who was a political failure, rather than a sixteenth century monarch who destroyed the signs of his own legitimacy." ${ }^{4}$ Jeux de pouvoir et jeux de langage semblent bien plus intimement liés que ne voudrait le laisser entendre une lecture orientée vers une conception du personnage du roi déchu sur un mode tragico-héroïque.

L'utilisation de l'ars rhetorica dans ce drame historique frappe le spectateur ou le lecteur par son degré de précision et d'à propos. Une étude fine et rigoureuse des structures rhétoriques utilisées par les divers

3. Wolfgang Clemen, The Development of Shakespeare's Imagery (London and New York: Methuen, 1951) 56.

4. Leonard Tennenhouse, Power on Display (London: Methuen, 1986) 81 . 
protagonistes du drame, ainsi que de leur agencement discursif, est un préalable indispensable à une compréhension précise de la pièce. Il importera toutefois de replacer la problématique du "souffle du roi" dans la perspective plus globale d'une compréhension du drame historique comme mode littéraire enchâssé dans le contexte socioculturel de l'Angleterre des Tudors.

La nécessité d'élargir l'étude rhétorique de l'œuvre à la notion plus large d'écriture dramatique de l'histoire de la nation, que l'on pourrait considérer également comme une mise en scène ou mise en drame de l'histoire et du politique, s'impose si l'on veut bien considérer la position de Richard II dans le Folio de 1623. Elle inaugure ce qu'il est coutume de désigner comme la deuxième tétralogic qui regroupe en une unité thématique trois pièces, dont une en deux volets, selon une cohérence thématique entérinée par la chronologie historique. Dans une relecture de la sćquence a posteriori, et sans préjuger de l'intention initiale de l'auteur, il nous semble permis de relever l'inversion des séquences historiques, puisque la première tétralogie, qui examine les règnes d'Henry IV et de Richard III, dépeint des événements postérieurs à ceux de la deuxième tétralogie. Le lecteur du Folio est invité à s'interroger sur une telle inversion, puisqu'elle figure bel et bien dans la version officielle du canon shakespearien. Ce qui frappe le lecteur à la faveur de cette recomposition chronologique, et ce depuis 1623, c'est une forme de mise en cause de la notion de chronique historique telle que pourrait la rendre l'épopée, par exemple, dans laquelle on insisterait simplement sur l'émergence de héros de l'histoire. À l'inverse, nous sommes confrontés à une forme de chronologie littéraire propre à rendre la volonté dramatique d'un homme de théâtre qui se saisit des ressources narratives que lui offre son propre patrimoine historique.

Il semble vain de relever les écarts littéraires que s'autorise Shakespeare par rapport à ses sources probables, les Chroniques de Holinshed, qui ne constituent somme toute qu'une forme de lieu mythique où se fondent éléments factucls et fiction. La cohérence littéraire qui justifie un agencement nouveau des faits de l'histoire s'exprime par le biais d'une réflexion sur la légitimité du monarque. Au travers de son Richard II, Shakespeare retrace la chute de la dynastie des Plantagenêts qui incarne une monarchie de droit divin caractérisée par le vieux Gaunt en ces termes sans appel adressés au roi Richard: "For thou art a king / But by fair sequence and succession?" (2.2.188-189). Cette chute porte au pouvoir la dynastie des Lancastre, caractérisée par un pragmatisme politique qui conduira ses divers représentants à lutter pour 
établir une légitimité fondée sur l'exercice juste du pouvoir, avec toujours à l'arrière plan la nécessité impérieuse d'effacer la faute originelle de la destitution du roi élu par Dicu. Le portrait du roi Henry $V$, monarque d'envergure aux vertus héroïques, mais également habile manipulateur des formes esthétiques du pouvoir, marque l'étape finale de cette quête d'une légitimité monarchique renouvelée, lorsque les vertus pratiques du gouvernant, révélées chez ce jeune monarque en situation de conflit armé, rachètent le nom des Lancastre entaché du sang d'un roi. La suprême ordalie de la guerre, qu'impose le divin au monarque, tel un contrepoint ironique à l'ordalie avortée qui initie le drame du roi Richard, établira aux yeux de tous la légitimité de celui que Dieu lui-même a élu et qu'il désigne comme son champion. Shakespeare utilise là une convention théologico-politique qu'il reprendra avec maestria dans l'épopée du fils miraculeux de l'usurpateur Bolingbroke. Ainsi se clôt le cycle thématique du questionnement de la légitimité monarchique inauguré par le drame du roi déchu, avec l'avènement d'un monde nouveau dans lequel la légitimitć monarchique se confond avec la virtu et non plus seulement la généalogie. Un monde dans lequel la quintessence de l'être rejoint en parfaite adéquation la forme visible.

Il n'y a donc pas lieu de s'étonner de l'inversion des données factuelles de l'histoire qu'opère le dramaturge, afin de clore sa séquence de drames historiques sur ce point d'orguc, à savoir le portrait d'un roi à la fois "lion et renard," selon les principes de Machiavel, un monarque qui sait conjuguer substance et valeur dans une orchestration experte des signes extérieurs qui désignent le monarque. En ce sens, la figure du roi Henry V s'apparente à celle de Richard III, qui marque l'étape finale de la première tétralogie, car tous deux sont d'habiles manipulateurs des signes visibles du pouvoir. Toutefois, l'issue de la première tétralogie, sans constituer une redondance thématique de la première, préfigure une ère de régénération politique, un renouvellement éthique annonçant l'ère nécessairement bénie du règne des Tudors. En tant que panégyriste du pouvoir en place, notre dramaturge semble ainsi souscrire au mythe Tudor. En toute bonne logique dramatique, Henry $V$ se clôt sur la célébration d'une union dans la plus pure tradition des comédies festives.

Si nous posons l'hypothèse de l'existence d'une unité dramatique qui régirait le développement des deux tétralogies, il convient d'envisager les notions de pouvoir et de théâtralité en termes de principes fondateurs d'une réflexion sur le politique dans les drames historiques de Shakespeare. De fait, par un effet spéculaire conventionnel, l'art de la mise en scène se révèle au cœur de la représentation de l'histoire dans la majorité des pièces historiques du Barde. Mettre en scène l'histoire équivaut à passer 
au crible de l'analyse le concept central du temps, car le théâtre possède cette aptitude intrinsèque à contracter ou à dilater le temps, voire à l'anéantir dans l'immédiateté de la représentation. Lorsque l'on parle de drame historique c'est bien à un jeu sur et avec le temps que l'on fait référence. Le théâtre de l'histoire dispose avant tout d'un temps historique, chronologique, événementiel qu'il modèle à l'aune du temps de la fiction dramatique et de la représentation scénique. Cette manipulation de l'événementiel est à considérer encore plus avant, car par le biais du miroir de la scène, par l'entremise des effets spéculaires dont le théâtre, de même que la représentation picturalc, a lc sccret, le drame historique peut établir un lien tangible entre le passé et le moment présent, entre l'Angleterre des Plantagenêts et celle de la rcine Élisabeth. L'alchimie temporelle de la représentation scénique, qui ouvre un espace contemporain à une fiction du passé, autorise la fusion entre passé et présent, entre histoire et chronique contemporaine. L'écriture théâtrale nous dévoile un autre élément de complexité, inhérent à sa nature, sous la forme de cette immédiateté de la représentation qui lui permet d'établir une tension entre ce qui prend corps sur la scène et le public qui assiste au spectacle. L'auditoire élisabéthain découvre dans le drame du roi destitué le reflet de son propre intérêt pour les rapports entre le pouvoir monarchique et la société dans son ensemble, un goût pour la mise en scène du politique que la reine Élisabeth manie avec un art consommé.

Toutefois, il nous semble essenticl de se garder de l'écucil qui consisterait à interpréter les drames historiques de Shakespeare comme des pièces à clef, faisant référence à des événements historiques contemporains de l'écriture, à l'instar de ceux qui se sont limités à lire dans le soulèvement de Bolingbroke une évocation de la rébellion du comte d'Essex. La mise en scène de l'histoire ne pcut se réduire simplement à l'élaboration d'unc œuvre codée qui permettrait d'aborder de façon voilée des thèmes auxquels on n'ose s'attaquer ouvertement. La mise en scène de l'histoire semble devoir être comprise comme un effet spéculaire de grande ampleur, réfléchissant au sens propre une problématique des formes théâtrales du pouvoir. Et la scène de théâtre présente toutes les qualités pour être perçue comme le lieu le plus approprié pour ce faire.

Il est permis de s'interroger sur ce choix générique d'écriture dramatique de l'histoire, que certains ont contesté pour la raison qu'il leur paraissait impropre à rendre un sujet qui, par nature, relèverait davantage de l'épopée, à tout le moins du mode narratif. Force est de constater que l'ampleur narrative et dramatique des deux tétralogies fournit un cadre propice à l'action du souffle épique caractéristique du traitement littéraire 
de l'histoire. Choisir l'histoire nationale comme source d'inspiration peut sembler un défi pour un dramaturge, la gageure d'imaginer une écriture dramatique autre que celle traditionnellement mise en cuvre pour des genres plus communément abordés au théâtre, tels la comédie, sous toutes ses formes, ou la tragédie. Cette ambiguïté de genre transparaît nettement dans le Folio de 1623 où l'on assiste à la création d'une catégorie distincte des deux précédemment citées, à savoir celle des "Histories." C'est pourquoi il nous paraît tout à fait légitime de nous interroger sur l'aptitude du mode dramatique à traiter une matière brute si peu orthodoxe. Ce problème central à notre propos nous conduit à réexaminer le "souffle des rois," la rhétorique historico-politique, en termes de théâtralité. ${ }^{5}$ L'ossature rhétorique si finement ouvragée de Richard II, évoquant à certains égards les principes de Castiglione au courtisan, nous apparaît comme une théâtralisation du verbe, une application des principes du decorum, une ornementation du langage parfaitement apparentéc à l'artificialité qui caractérise la vie de Cour. ${ }^{\circ}$

L'examen des formes théâtrales du pouvoir telles qu'elles sont présentées dans Richard II, invite à se replacer dans une problématique du pouvoir monarchique propre à la Renaissance, que Kantorowicz désigne sous le vocable "les deux corps du Roi." Cette dualité de la personne royale, telle qu'on la conçoit à la Renaissance, fonde l'existence d'une créature mythique, une "persona ficta" ${ }^{7}$ qui incarne au plein sens du terme la monarchie. S'il y a gémellité, il y a également fusion des deux corps qui forment une unité indivisible, avec cette réserve que lc corps politique transcende l'enveloppe corporelle du monarque. ${ }^{8}$ De fait, cette fiction de l'unité des deux corps élabore une représentation mythique de la personne physique du monarque car celui-ci manifeste par sa corporalité la

5. Le théâtre du politique est avant tout une réflexion sur le politique de manière atemporelle. Cependant, la mise en lumière du théâtre du pouvoir par le modeste théâtre des hommes, dans lequel les monarques deviennent de simples "mechanics," ne peut manquer de réfléchir cette image, à la manière d'un miroir géant, sur le pouvoir politique en place, participant par là même à cette mise en scène à grande échelle. Le théâtre met à $\mathrm{nu}$, réfléchit, dénonce. Propagande ou subversion sont les deux extrêmes entre lesquels oscille le théâtre du pouvoir. La problématique spéculaire du regard qui voit tout en se donnant à voir fera l'objet de toute notre attention dans cette étude du roi destitué.

6. Baldassare Castiglione, The Book of the Courtier, 1561, trad. Sir Thomas Haboy, (London and Toronto: J. M. Dent \& Sons, 1908).

7. Ernst Kantorowicz, The King's Two Bodies: a Study in Medicval Theology (Princeton: Princeton UP, 1957) 19.

8. Kantorowicz 23: "le corps naturel, par cette conjonction du corps politique avec lui ... est magnifié, et par cette même consolidation, il contient en lui le corps politique." 
symbiose entre le pouvoir que lui confère son état de dirigeant politique légitime, d'une part, et son enveloppe charnelle, d'autre part. La nécessité pour le politique de se mettre en scène, d'offrir son corps magnifié aux regards de tous, traduit l'impératif esthétique du politique, que Leonard Tennenhouse analyse au regard de l'immanence du pouvoir exercé. ${ }^{9}$

Comprendre l'importance du spectacle royal, c'est assurer la stabilité de la monarchie. La faute première de Richard II réside dans son incompétence à manier l'art du spectacle avec à propos. Il rejette les accessoires du pouvoir pour la raison qu'il choisit de retenir seulement dans la fiction du corps politique sa dimension de principe établi et incontestable, d'où découle, selon lui et selon l'ordre médiéval désormais caduc, l'invulnćrabilité du monarque. Le dernier des Plantagenêts devient par voie de conséquence la victime d'une fiction qu'il interprète en termes cosmiques et sacramentels: "Not all the water in the rough rude sea / Can wash the balm off from an anointed king" (3.2.54-55). Il néglige ainsi totalement la dimension artificielle mais néanmoins fondamentale du spectacle royal.

La théâtralisation de la monarchie, indispensable à l'incarnation du corps politique, se traduit au plan langagier par l'élaboration d'une esthétique du verbe, propre à accompagner l'esthétique du politique. Cette esthétique du verbe recouvre une utilisation savante de l'art de persuader, dont la fonction est associée par Stcphen Greenblatt à l'art dramatique: "[to] theatricalize culture." ${ }^{10}$ La Cour du roi, ce haut lieu de théâtralité, constitue la scène où le politique se donne en perpétuelle représentation, aussi bien visuelle que linguistique. Se trouve visiblement modifiée au cours des siècles, avec la succession des différents monarques, la place dévolue à l'esthétique du pouvoir, donc au regard porté sur l'enveloppe charnelle du souverain. Shakespeare en atteste dans ses portraits de monarques, depuis Édouard III jusqu'à Henry V.

Le monde féodal, incarné par Édouard III et représenté dans Richard II sous les traits de Gaunt et York, met en avant la nécessité d'une adéquation entre aspect physique, dignité royale et vertu. York relève une forme d'ironie dramatique lorsqu'il souligne l'absence d'adéquation entre l'apparence du roi, qui rappclle celle de son père, et son être intime, qui manque singulièrement d'étoffe:

9. Tennenhouse 105: "In a system where the power of the monarch was immanent in the official symbols of the state, the natural body of the monarch was bound by the same poetics of display."

10. Stephen Greenblatt, Renaissance Self-Fashioning; from More to Shakespeare (Chicago : U of Chicago P, 1980) 162. 
His face thou hast, for even so look'd he, Accomplish'd with the number of thy hours;

But when he frown'd it was against the French, And not against his friends. . . (2.1.176-180)

Cette remarque n'est pas sans importance car elle résume une problématique fondamentale de la pièce, celle des relations entre l'être et le paraître. Si le Moyen Âge est présent dans Richard II au travers de toutes les références à l'allégeance féodale des vassaux à leur souverain, rappelée dans la scène 1 de l'acte I par. les formules rituelles qu'emploient les plaignants, sa relation au langage semble déjà archaïque. À l'inverse, Shakespeare nous montre qu'un changement profond s'est fait jour au plan épistémologique dès lors qu'il n'y a plus adéquation entre signe et sens. Le monde des signatures, tel que décrit par Michel Foucault dans Les Mots et les choses, cède le pas devant l'émergence du couple signifiantsignifié et plus particulièrement devant l'effacement progressif du signifié au profit du signifiant." En vertu de cette évolution, il semble que les personnages portent une plus grande attention à la forme du discours, qui désormais sert davantage à masquer qu'à exprimer. Les figures du secret supplantent celles d'une énonciation directe et franche. En insistant sur la misc en scène du discours, en élaborant les formes rhétoriques comme l'hyperbole, on creuse davantage le fossé entre le signe et son référent. Le verbe cesse d'être un instrument de communication directe entre locuteurs. À l'inverse, il se replic sur lui-même et fonctionne sans relation directe à un référent quelconque, à l'instar d'une mécanique autonome.

L'intérêt pour les formes esthétiques s'accroît et dans un même temps le travestissement du verbe s'ćlabore. Les modes allégorique, symbolique, emblématique, dont l'aptitude mimétique était fort prisće des rhétoriciens médiévaux, cèdent le pas devant une rhétorique de l'hyperbole et de l'ambiguité. Travestir ce que l'on souhaite dire devient la préoccupation majeure de la Cour. Ce phénomène d'évolution du signe, de la prépondérance du signifié vers une importance grandissante du signifiant, repose sur dcux concepts fondamentaux de la Renaissance anglaise: le premier est de nature linguistique, le second relève de la philosophie appliquée au fonctionnement social.

Le concept linguistique au cœur d'une mise en scène du verbe de plus en plus élaborée est celui de la réactivation des signes comme entités distinctes de leur référent, par conséquent modelables à souhait. Lars Vissing donne une analyse précise de ce fonctionnement dans son ouvrage sur Machiavel:

11. Michel Foucault, Les Mots et les choses (Paris: Gallimard, 1966) 32-60. 
Alors que le langage avait été l'instrument adéquat et complet de l'expression de la réalité, que nommer une chose, c'était déjà l'expliquer, que l'opposition entre les mondes respectivement célestes et terrestres n'était pas évidente, que l'ensemble de ces faits rendait malaisée la distinction des parts respectives de l'abstrait et du concret dans l'outillage mental du Moyen Âge, la Renaissance amène la restauration de la fonction représentative du signifiant et, par là, également, les conditions d'une antinomie res/verba. ${ }^{12}$

Il poursuit en citant Le Goff: "Ce regard s'arrête désormais sur les apparences et, au licu d'être un simple symbole de la réalité cachée, le monde sensible prend valeur en soi, est objet de délectation immédiate." 13 Le signe, qu'il soit visuel ou linguistique, prend désormais de l'importance en tant qu'élément autonome. Il gagne donc en visibilité immédiate. Par voie de conséquence, si l'on postule que le signe existe en tant qu'entité dissociable de son référent, si la fonction mimétique du verbe ne peut plus être envisagée en tant que fondement langagier, il devient alors possible de dévier celui-ci de son utilisation originelle, de le pervertir, et de le laisser fonctionner à vide, telle une simple enveloppe. Ceci ne laisse pas d'être inquiétant, si l'on songe au transfert métaphorique possible entre ce qui définit le signe linguistique et le signe visucl, entre la production d'un discours et la mise en scène du monarque, entre l'enveloppe vide du langage et ce que Shakespeare appelle l'ombre d'un roi. Si le verbe est capable de fonctionner sans référent, faudrait-il alors en déduire que le rôle du monarque peut être tenu sans que s'établisse un lien avec aucune substance, comme cela se voit communément au théâtre où des "mechanics" peuvent être rois?

L'autre concept fondateur qui relève de l'évolution vers une attention croissante portće aux formes esthćtiques du pouvoir constitue ce que Stephen Greenblatt nomme "fashioning." Ce concept désigne ce qui a été analysé parfois en termes de paraître machiavélien, à savoir le dispositif de techniques illusionnistes du pouvoir tel que le décrit Machiavel dans Le Prince. Des termes comme "fashioning" ou "the forming of a self" définissent cette pratique de modelage de l'image, donc de l'identité sociale, qui caractérise la Cour à la Renaissance. Stephen Greenblatt s'inspire de l'analyse de Burckhardt pour justifier l'apparition de ce phénomène nouveau, en Italie:

the transition form feudalism to despotism fostered a radical change in consciousness; the princes and the condottieri, and their secretaries,

12. Lars Vissing, Machia vel et la Politique de l'Apparence. (Paris: PUF, 1986) 29.

13. Vissing 29. 
ministers, poets and followers, were cut off from established forms of identity and forced by their relationship to power to fashion a new sense of themselves and their world: the self and the state as works of art." ${ }^{14}$

Le changement de cadre épistémologique au sens large invite à une reconstruction identitaire pour tous les acteurs de la société. À cette occasion, le paraître prend le pas sur l'être, au point de le supplanter. La conscience chez le monarque de la nécessité de façonner une identité nouvelle, après l'anéantissement des absolus médiévaux, met en lumière l'importance accordée au masque social. D'où l'apparition de guides à l'attention du courtisan, visant à facilitcr par des conseils pratiques la mise en scène de l'homme de Cour et offrant ce que Greenblatt nomme "a model for the formation of an artificial identity." ${ }^{15}$ Castiglione est à l'origine du plus célèbre de ces manuels, connu en Angleterre sous le titre The Book of the Courtier. Cet ouvrage offre des stratégies de dissimulation et de feinte, en matière rhétorique notamment. Il s'apparente en tous points à un manuel pratique pour comédien. L'usage consommé de la rhétorique constitue un facteur essentiel dans l'élaboration de ces stratégies de façonnement d'une identité fictive car il participe de la mise en scène de l'individu dans un monde d'apparences.

L'importance croissante du trompe-l'œil comme principe fondateur du comportement social et de l'exercice du pouvoir, est parfaitement en accord avec les principes machiavéliens du paraître. Lars Vissing résume cela par les expressions "montrer tout en dissimulant," ou bien jouer "d'effets de surface," ${ }^{6}$ ce qui consiste à faire preuve d'illusionnisme politique en réponse à l'attente de l'ensemble des sujets, et non par penchant tyrannique. Telles sont en substance les recommandations de Machiavel au Prince. En d'autres termes, l'accent est très nettement déplacé vers le signifiant qui se dissocie désormais de son référent. Shakespeare nous donnera une illustration de cette technique dans la tactique adoptée par Henry IV puis Henry $\mathrm{V}$ afin d'assurer leur pouvoir. La permanence mythique et symbolique du Moyen Âge se trouve bien révolue lors de l'avènement de ces praticiens de théâtre qui savent joucr du paraître. Le langage utilisé par les différents personnages d'une pièce comme Richard II, exprimant diverses positions à l'égard de l'esthétique du pouvoir, est le plus sûr marqueur de ces "effets de surface."

14. Greenblatt 161 .

15. Greenblatt 162.

16. Vissing 37, 207. 
Afin d'ouvrir le volet consacré au reflet trompeur, laissons retentir la voix qui vient du passé, celle qui incarne le monde féodal en déclin et dont les prédictions poursuivent le souverain, en un mot la voix du vieux Gaunt. Sur son lit de mort, l'oncle du roi singe l'art du courtisan en jouant sur la réversibilité du verbe, comme il l'a vu faire maintes fois par ceux qui ont l'oreille du roi et savent le charmer à l'aide de ce que Gaunt qualifie de "lascivious metres" (2.1.19):

$\mathrm{O}$, how that name befits my composition!

Old Gaunt indeed, and Gaunt in being old.

Since thou dost seek to kill my name in me,

I mock my name, great king, to flatter thee! (2.1.73-74, 86-87)

Ce premier assaut verbal, sur un mode délibérément ironique puisqu'il vise à mettre au jour la réversibilité, donc la vacuité, du langage de Cour, se poursuit par un rapide échange stichomytique, illustrant à son tour la notion de réversibilité au moyen d'une antimétabole où vie et mort se révèlent interchangeables:

RICHARD. Should dying men flatter with those that live

GAUNT. No, no, men living flatter those that die.

RICHARD. Thou now a-dying sayest thou flatterest me.

GAUNT: Oh, no, thou diest, though I the sicker be.

RICHARD. I am in health, I breathe and see thee ill.

GAUNT: Now He that made me knows I see thee ill,

Ill in myself to see, and in thee, seeing ill. (2.1.88-94)

L'enchaînement des antimétaboles, avec le renversement paradoxal du vers 91 , se clôt sur une épanalepse au vers 94, qui produit un effet spéculaire saisissant d'à propos. L'accumulation d'antimétaboles, utilisés comme marqueurs de la notion de réversibilité, trouve son apothéose quelques vers plus loin, dans une formule lapidaire qui résume parfaitement la trame narrative du drame: "Deposing thee before thou wert possess'd, / Which art possess'd now to depose thyself" (2.1.107-108). La réversibilité subversive du pouvoir, telle que la dévoile Gaunt, désigne avant tout la réversibilité du signe, en tant que surface visible, dont les qualités protéennes peuvent conduire le monarque peu averti à se perdre dans le reflet mystificateur que lui renvoic le miroir des flattcurs. N'oublions pas, à ce propos, que Richard choisit de fairc usage d'un miroir en tant qu'accessoire de scène lors de la représentation de ce que Shakespeare nomme le "woeful pageant" de sa destitution (4.1.320), ou de ce que l'on pourrait baptiser l'antimasque du pouvoir.

De fait, les miroirs linguistiques sont cumulatifs car ils suivent de près le déroulement de la diégèse, ou plutôt l'effet de balancier que 
produit le cheminement des deux personnages principaux, en accord avec le mouvement de la "Roue de la Fortune": Richard, tel Phaéton, seau vide néanmoins plein de larmes, et Bolingbroke, l'usurpateur, semblable au soleil du matin en sa course ascendante.

L'effet optique de réversibilité du pouvoir est tout d'abord rendu en faisant appel au registre visuel, par l'image des deux protagonistes tenant chacun la couronne d'une main, tout en se faisant face. Ils donnent ainsi l'illusion visuelle que l'un n'est que le reflet de l'autre. Cet effet spéculaire a inspiré l'imagination de certains metteurs en scène, qui ont choisi de prolonger cet effet en demandant aux deux protagonistes du pouvoir d'intervertir leurs rôles un soir sur deux, après un tirage au sort préliminaire à chaque début de représentation. ${ }^{17}$

La duplication visuelle, incarnation du procédé de l'antimétabole qui traduirait la réversibilité du pouvoir, se trouve prolongée dans la même scène par le premier vers du discours de désacralisation du monarque: "Ay no; no ay." (4.1.200). Cette antimétabole d'une concision rare offre un écho linguistique à l'image, l'emblème peut-être d'un pouvoir fracturé, du corps du roi scindé en deux entités distinctes. La thèse de la fracture se trouve même confortée si l'on choisit d'entendre: "I no; no I; for I must be nothing." De fait, une telle interprétation viendrait appuyer l'idée du divorce du corps du roi, d'une enveloppe désormais privée de substance, que contribuerait à actualiser le contre-rituel qui suit immédiatement. S'il est anachronique de faire appel à la notion de schizophrénie ici, il reste cependant possible de parler de dichotomie du corps royal, mise en scène dans l'épisode du miroir. Nous assistons à l'apparition du "mockery king of snow," (4.1.260) voué à se dissoudre à la chaleur des rayons du nouveau monarque; cette image de "non-entité" par effet de liquéfaction confirme le divorce des deux corps d'un roi réduit à une enveloppe privée de substance, littéralement l'ombre d'un roi. Le bris du miroir, la dissémination du visage royal, cède la place au néant.

L'art de la réversibilité, pratique courtisane dénoncée par le vieux Gaunt, est un principe fondateur de la rhétorique de Cour, telle qu'elle apparaît dans une scène de règlement de justice aussi ritualisée que celle qui ouvre la pièce. Le monarque, incarnation du pouvoir divin, revêt les attributs de sa fonction d'arbitre et se propose d'entendre publiquement les adversaires: "the accuser and the accusèd freely speak" (1.1.17) En réalité cette formule codifiée est aussi trompeuse que la rhétorique qu'il s'apprête à entendre chez les deux partis en présence, tout comme peuvent l'être les

17. Juhn Barton, avec Richard Pasco et Ian Richardson, Royal Shakespeare Company, 1973. 
"lascivious metres" des courtisans, manipulateurs de verbe. Preuve en est l'inflation hyperbolique de cette scène placée sous le signe de l'exagération coupable. Celui dont le langage est le plus hyperbolique se révèle être le coupable, car l'écart entre le signe et son référent est de facto maximal: "We thank you both, yet one but flatters us, / As well appeareth by the cause you come (1.1.25-26)." L'ambiguité produite par l'usage de l'amphibologie aux vers 132 à 134 et 135 à 137, désigne un langage qui tourne à vide. Cette langue apparaît dès lors comme figée dans une raideur dogmatique; elle se perd en stéréotypes langagiers et représente plus qu'clle n'exprime.

La prétérition par laquelle Mowbray annonce qu'il se gardera d'utiliser le verbe comme une arme marque ses propos d'une empreinte rhétorique médiévale:

Let not my cold words here accuse my zeal.

'Tis not the trial of a woman's war,

The bitter clamour of two eager tongues,

Can arbitrate the cause betwixt us twain. (1.1.46-50)

Sa fonction consiste en réalité à masquer l'acte d'accusation véritable, lancé à l'encontre du souverain, tout en le laissant filtrer à mots couverts. Le code verbal extrêmement rigide qui caractérise l'esprit chevaleresque, laisse transparaître, au moment où il dévoile aux spectatcurs son artificialité, la nature véritable de l'accusation, ainsi que l'identité de son destinataire. La parodie d'ordalie, interrompue précocement par un geste d'arbitraire royal, signe la culpabilité du roi qui tente d'entraver le cours du langage, de pervertir sa fonction de communication. La tyrannie royale qui s'exprime alors consiste en un travestissement du langage mis cn acte, ou, dans une version métaphorique, la transformation de l'organe de la langue en un battant de cloche qui ne résonne plus:

And now my tongue's use is to me no more

Than an unstringed viol or a harp,

Or like a cunning instrument cas'd up-

Or being open, put into his hands

That knows no touch to tune the harmony.

Within my mouth you have engoal'd my tongue. . . (1.3.161-166)

Ce que nous pourrions considérer comme la forme du silence sćmantique d'un langage qui se donne à entendre, est la conséquence aussitôt perceptible d'un pouvoir qui a glissé vers la tyrannie. La théatralisation du langage par le biais de la rhćtorique fonde le pouvoir, à la condition qu'elle maintienne une certaine souplesse linguistique qui autorise la 
communication verbale. Une langue qui, ne parvenant plus à exprimer de contenu sémantique, se replie sur sa seule dimension formelle, exerce, si l'on peut dire, une tyrannie de la forme.

Un dernier signe de gauchissement du langage, comme reflet d'une théâtralisation mal comprise du pouvoir, réside dans l'exigence d'une réécriture de l'histoire que formule le roi déchu sur le seuil de son cachot. Il revendique une mise en scène subversive de la diégèse historique a posteriori. Il serait tentant de voir dans cet épisode un effet spéculaire du dramaturge sur sa propre activité, révélant la nature potentiellement subversive de sa fonction:

In winter's tedious nights sit by the fire

With good old folks, and let them tell thee tales

Of woeful ages long ago betid;

And ere thou bid good night, to quite their griefs

Tell thou the lamentable tale of me,

And send the hearers weeping to their beds;

For why, the senseless brands will sympathize

The heavy accent of thy moving tongue,

And in compassion weep the fire out,

And some will mourn in ashes, some coal-black,

For the deposing of a rightful king. (5.1.40-50)

Ce repositionnement de la figure royale déchue au niveau mythique, dans le flot de l'histoire où les rois survivent sous forme de légende, est un exemple de la manipulation de la diégèse au moyen de termes linguistiques reposant sur l'effet mystificateur de la fable. Notons que cette réécriture gauchie de l'histoire, familière aux tenants de la dictature, sert à merveille le roi déposé. En effet, c'est le souvenir de sa prestation subversive dans la pièce intitulée "The Beggar and the King" (4.1), enchâssée dans l'œuvre principale, qui permet à Richard de peser de tout son poids dans le seau métaphorique qu'il évoque, faisant ainsi monter celui du nouveau monarque. En vertu des lois les plus élémentaires de la physique, ne pourrait-on considérer que, celui des deux seaux qui descend étant le plus lourd, c'est véritablement le personnage incarnant la subversion des modes esthétiques du pouvoir, et révélant sa nature même d'acteur, qui a le plus grand impact dramatique? Bien entendu, ce talent s'exprime au détriment de l'exercice et de la possession du pouvoir. Toutefois, c'est dans l'économie de la pièce de Shakespeare qu'il atteint la force de conviction la plus grande. Il est certes un excellent acteur dans le théâtre des hommes; mais c'est pour cette raison précise qu'il s'éloigne de son rôle initial d'acteur du pouvoir. Une telle dichotomie, au cœur même du drame, sera résolue dans ce que nous pourrions baptiser le théâtre du 
pouvoir Tudor, théâtre des hommes et théâtre de Cour: la fusion des "deux corps de l'acteur" pourra enfin s'opérer.

Ainsi que nous l'avons vu, le drame du roi déposé manifeste une dissociation entre le roi comédien, capable d'émouvoir un auditoire hors du cadre du drame, et le roi acteur du pouvoir, qui ne parvient pas à exécuter son contrat de théâtralité. Le roi déchu cède la place à d'habiles manipulateurs de la forme théâtrale, fût-elle royale ou littéraire. Celui qui représente le mieux cette tendance est Henry V, dont l'épopée clôt le second volet des drames historiques. Un premier exemple de son adresse à utiliser l'esthétique théâtrale réside dans le soin qu'il met à dramatiser son entrée de souverain resplendissant. Il l'annonce clairement mais a le souci de la différer. Le goût qu'a le prince Hal pour le déguisement lui permet de retarder tout au long du règne de son père la révélation de son étoffe royale. À l'aune du temps dramatique, cet intervalle couvre les deux volets d'Henry $I V$. Le souverain autrefois rival de Richard II croit ainsi reconnaître en son propre fils les traits "défigurés" de son prédécesseur. Sa profession de foi, le futur Henry V nous la donne d'emblée, sous la forme d'un soliloque, afin qu'il soit bien établi que nous sommes en présence d'un manipulateur de signes, à son affaire lorsqu'il s'agit de se mettre en scène:

Yet herein will I imitate the sun,

Who doth permit the base contagious clouds

To smother up his beauty from the world, That when he please again to be himself, Being wanted he may be more wondered at

So when this loose behaviour I throw off

And pay the debt I never promised

By how much better than my word I am,

By so much shall I falsify men's hopes;

And like the bright metal on a sullen ground, My reformation, glitt'ring o'er my fault,

Shall show more goodly and attract more eyes. (1 Henry IV, 1.2.[194-214])

La théâtralité du pouvoir à laquelle le futur monarque fait ici référence s'avérera, comme l'on pouvait s'y attendre, en harmonie avec l'étoffe royale qui est la sienne.

La nouvelle génération de monarques, conscients que le spectacle est le principe fondateur de leur pouvoir effectif sur l'imaginaire de leurs sujets, nous conduit tout naturellement à la dynastie des Tudors, et plus particulièrement à la reine Élisabeth. Précisons toutefois que, selon les 
termes mêmes de ce qu'il est convenu d'appeler le "mythe Tudor," le spectacle du pouvoir semble ici bien compris et ne constitue en aucune façon un leurre susceptible de masquer des pratiques peu orthodoxes. Dans l'œuvre même de Shakespeare, il est possible d'observer cette évolution à l'occasion de la rédaction plus tardive de la pièce intitulée $A l l$ is True (Henry VII). Elle fait intervenir des formes esthétiques codées, tel le masque, afin de manifester la théâtralité du monarque et de sa Cour. En effet, le recours au masque qui constitue une forme théâtrale fortement marquée, au sein même d'un drame historique centré sur l'examen du pouvoir, marque la symbiose possible entre les acteurs du pouvoir et les comédiens qui officient généralement sur les scènes de théâtre. De plus, la forme dramatique du masque, associée de manière générale à la mise en scène d'une splendeur et d'une harmonic divine, peut certainement traduire en termes esthétiques l'ordre régnant dans le royaume. Elle pourrait même faire office d'organe de propagande pour le pouvoir en place, entre les mains du dramaturge. On rappellera, à ce propos, la pratique qui consistait à réserver les rôles principaux de ces masques au monarque et autres dignitaires de la Cour, produisant de cette manière un effet spéculaire tout à fait saisissant de l'acteur roi, comédien en scène.

La reine Élisabeth, qui nous intéresse au premier chef car elle est contemporaine de l'écriture des drames historiques, nous offre un exemple remarquable de la stratégie du politique interprété en termes esthétiques, que Donald Watson qualifie de "aestheticizing politics." 18 Il est de notoriété publique qu'Élisabeth cultivait l'art de transformer en spectacle chacun de ses déplacements, notamment ses "summer progresses," et se désignait comme le premier sujet d'inspiration de la littérature et de l'iconographie de l'époque. Donald Watson analyse très précisément ce phénomène:

Her incarnation as Diana, Cynthia, Angelica, Gloriana, Astraea, Britannia, and the Virgin Queen cost her nothing and won her the praises of poets and citizens alike. The rhetoric of monarchy used all the arts of display to present the Queen as the only actress on the stage of politics. . . the outer circles of the Court of Elizabeth were theatricalized by the revival of chivalry, the acculturation of European conventions of courtly behavior, and the prevalence of Petrarchan politics, that is, the translation of attendance at Court into erotic metaphors of serving an unattainable mistress. ${ }^{19}$

18. Donald G. Watson, Shakespeare's Early History Plays: Politics at Play on the Elizabethan Stage (London: Macmillan, 1990) 23.

19. Watson 23 
L'appartenance de ce monarque au sexe féminin modifie, de toute évidence, les données du problème. Cela favorise certainement une utilisation encore plus poussée des formes esthétiques du pouvoir. Elles garantissent la stabilité monarchique menacée par l'éventualité d'un mariage qui conduirait la reine à aliéner sa souveraineté à son légitime époux.

Ce qui demeure inchangé, c'est bien cette volonté d'effacer la distinction entre la scène du théâtre et la scène de l'État: "politics is play, politics as play, and the play with politics: the stage is all the world." 20 De fait, le théâtre élisabéthain devient, précisément pour cettc raison, la scène privilégiée de la royauté, qui aime à se voir représentée - ce qui conforte son existence - dans le cadre de drames historiques ou de comédies où l'on dépeint la nécessité d'un retour à l'ordre. Le spectacle du pouvoir représenté sur la scène d'un théâtre est, au-delà du risque d'un effet de subversion, un formidable instrument de consolidation politique.

Marie-Christine MUNOZ

Université Paul Valéry-Montpellier III

20. Watson 151 . 